\title{
Ion irradiation of multi-walled boron nitride nanotubes
}

\author{
Ossi Lehtinen ${ }^{\star}, 1$, Timur Nikitin ${ }^{2}$, Arkady V. Krasheninnikov ${ }^{1,3}$, Litao Sun ${ }^{4}$, Leonid Khriachtchev ${ }^{2}$, Florian \\ Banhart $^{5}$, Takeshi Terao ${ }^{6}$, Dmitri Golberg ${ }^{6}$, and Juhani Keinonen ${ }^{1}$ \\ ${ }^{1}$ Division of Materials Physics, University of Helsinki, P.O. Box 43, 00014 Helsinki, Finland \\ ${ }^{2}$ Laboratory of Physical Chemistry, University of Helsinki, P.O. Box 55, 00014 Helsinki, Finland \\ ${ }^{3}$ Laboratory of Physics, P.O. Box 1100, Helsinki University of Technology, 02015 Helsinki Finland \\ ${ }^{4}$ Key Laboratory of MEMS of Ministry of Education, Southeast University, Nanjing 210096, China \\ ${ }^{5}$ Institut de Physique et Chimie des Matériaux de Strasbourg, IPCMS-DESI, UMR 7504, 23 rue du Loess, 67034 Strasbourg, France \\ ${ }^{6}$ World Premier International Center for Materials Nanoarchitectonics (MANA), National Institute for Materials Science, Namiki 1-1, \\ Tsukuba, Ibaraki 3050044, Japan
}

Received 29 May 2009, revised 3 November 2009, accepted 16 December 2009

Published online 4 February 2010

PACS 61.72.Cc, 61.80.Jh, 81.07.Dc

* Corresponding author: e-mail ossi.lehtinen@helsinki.fi, Phone: +358-9-19150018, Fax: +358-9-19150042

Multi-walled boron nitride nanotubes were irradiated with low and medium energy argon and helium ions at room and elevated temperatures. The irradiated samples were characterized by transmission electron microscopy and Raman spectroscopy, and a comparison to the response of carbon nanotubes to irradiation was made. A dose of $2 \times 10^{15}$ ions $/ \mathrm{cm}^{2}$ was found to give rise to complete amorphization for irradiation with $40 \mathrm{keV}$ Ar ions, while a comparable dose (in terms of displacement per atom) of $1.2 \times 10^{18}$ ions $/ \mathrm{cm}^{2}$ for $350 \mathrm{keV} \mathrm{He}$ caused significantly less damage. Elevated temperatures considerably reduce the amount of damage indicating that efficient annealing of defects occurs in $\mathrm{BN}$ nanotubes already at $300^{\circ} \mathrm{C}$. Our results provide evidence that multiwalled BN nanotubes have similar or even better stability under ion irradiation than their carbon counterparts.
1 Introduction Lots of research has recently been done on technologically important $\mathrm{sp}^{2}$-bonded carbon and boron nitride nanostructures. In these systems, the atoms are arranged in a similar honeycomb lattice, but in BN nanotubes [1,2], fullerenes [3] and single BN sheets [4] alternating $\mathrm{B}$ and $\mathrm{N}$ atoms substitute for $\mathrm{C}$ atoms. Mechanical properties of $\mathrm{BN}$ and $\mathrm{C}$ nanotubes are of the same impressive order, but BN tubes possess higher thermal and chemical stability [5].

Interaction of energetic particles such as electrons [68] and ions [9-11] with nanostructures has recently been in the focus of research as well. This subject is interesting from both fundamental and practical points of view: the nano-size of such systems and quantum-mechanical size quantization effects give rise to new interesting phenomena which can be used for engineering the atomic and electronic structure of nanosystems [12]. With regard to the response of $\mathrm{BN}$ nanosystems to electron irradiation, the experiments have shown somewhat higher resilience of
BN multi-walled nanotubes (BN MWNTs) as compared to carbon multi-walled carbon nanotubes (C MWNTs) [13], which is an unexpected result as simulations have predicted lower displacement energies for both $\mathrm{B}$ and $\mathrm{N}$ atoms in $\mathrm{BN}$ nanotubes as compared to carbon atoms in carbon nanotubes [14]. Moreover, low-dose irradiation was reported to even give rise to the annealing of defects formed during the growth of BN MWNTs [15]. It was also demonstrated that electron irradiation of BN MWNTs at low (104 K) temperatures does not give rise to amorphization after considerable irradiation [8]. Based on this result, the authors argued that the damage and the annealing mechanisms in $\mathrm{BN}$ nanotubes is different from that of carbon nanotubes where the sample temperature is a major factor in irradiation response.

While there is some experimental, though not fully understood, data on the response of $\mathrm{BN}$ nanotubes to electron irradiation, there is essentially no experimental information on ion irradiation of $\mathrm{BN}$ nanotubes. Relatively little 
research has been conducted so far on bulk BN systems [16], and it is not obvious at all if the data received for bulk phases is relevant to $\mathrm{BN}$ nanosystems.

In this paper we present the results of our experiments on ion irradiation of $\mathrm{BN}$ nanotubes. Also a comparison to the response of C MWNTs to ion irradiation was made.

\section{Experimental details}

2.1 Samples MWBNNT were ultrasonically dispersed in ethanol and a few drops of the resultant solutions were dripped on standard $3 \mathrm{~mm}$ TEM grids coated with a thin carbon film. The procedure ensures uniform distribution of the nanotubes over the grids and provides a homogeneous nanotube film (randomly oriented tubes) of approximately one nanotube diameter thickness. The nanotubes on the grids have typical diameters of 20-70 $\mathrm{nm}$ and did not have any $\mathrm{BN}$ or $\mathrm{B}_{2} \mathrm{O}_{3}$ particles which can sometimes be found in the as-grown material [17].

2.2 Irradiation The samples were irradiated using a $500 \mathrm{kV}$ ion implanter. Source gas (Ar and $\mathrm{He}$ ) was ionized in a cold cathode penning ion source and the ions were accelerated with an electrostatic field generated with a driver unit and a multi-doubler stack. The produced focused ion beam was run through a beam sweeping system which rasterized the beam over selected target area to produce a homogeneous irradiation treatment.

The ion beam heated the sample in case of He ions. A rough upper estimate of the resulting temperature is given in Table 1. As the contact area between nanotubes and the grid is unknown, the equilibrium temperature was calculated taking into account only power coming in via the ion beam and power going out through thermal radiation. Thus the true temperatures were lower than the estimated upper limit. Sample d was heated up to the given temperature with an heatable sample holder and the temperature estimate should be more accurate.

2.3 High-resolution transmission electron microscopy The BNNT samples were studied using a $300 \mathrm{kV}$ HR field-emission TEM JEM3100FEF (JEOL) equipped with in-column Omega Filter. Carbon nanotube samples were examined in a HR-TEM FEI Tecnai F-30 with a field emission gun and an acceleration voltage of $300 \mathrm{kV}$.

Table 1 The boron nitride samples tabulated. The sample column corresponds with the panel labeling in Fig. 2. $\phi$ stands for irradiation dose.

\begin{tabular}{lllllll}
\hline Smpl. & Ion & $\mathrm{E}(\mathrm{keV})$ & $\phi\left(1 / \mathrm{cm}^{2}\right)$ & dpa & $\mathrm{T}(\mathrm{K})$ & est. T (K) \\
\hline $\mathrm{a}$ & $\mathrm{Ar}$ & 40 & $1 \times 10^{14}$ & 0.13 & $\mathrm{RT}$ & $\sim \mathrm{RT}$ \\
$\mathrm{b}$ & $\mathrm{Ar}$ & 40 & $5.5 \times 10^{14}$ & 0.7 & $\mathrm{RT}$ & $\sim \mathrm{RT}$ \\
$\mathrm{c}$ & $\mathrm{Ar}$ & 40 & $2 \times 10^{15}$ & 2.6 & $\mathrm{RT}$ & $\sim \mathrm{RT}$ \\
$\mathrm{d}$ & $\mathrm{Ar}$ & 40 & $2 \times 10^{15}$ & 2.6 & 900 & $\sim 900$ \\
$\mathrm{e}$ & $\mathrm{He}$ & 350 & $3.5 \times 10^{16}$ & 0.06 & 300 & $\sim 400$ \\
$\mathrm{f}$ & $\mathrm{He}$ & 350 & $1.2 \times 10^{18}$ & 1.9 & 300 & $\sim 600$ \\
\hline
\end{tabular}

2.4 Raman spectroscopy The Raman measurements were carried out using a single-stage spectrometer (Acton SpectraPro 500I, resolution $10 \mathrm{~cm}-1$ ) equipped with a charge-coupled device camera (Andor InstaSpec IV) and an Ar+ laser (Omnichrome 543-AP, $100 \mathrm{~mW}$ at $514.5 \mathrm{~nm}$ ). The laser radiation was focused to the sample surface, and Raman light was detected in the transverse direction without polarization analysis. More details of the experimental procedures can be found elsewhere [18]. As BN nanotubes are wide-gap semiconductors [5], one can expect that the whole volume of the nanotubes was probed by Raman spectroscopy.

\section{Results and discussion}

3.1 Displacements per atom As displacement per atom (dpa) is an important quantity to assess the amount of damage created by the ions, computer program TRIM [19] was used to estimate the amount of damage resulting from different irradiation treatments. In fact dpa gives the upper limit for the amount of damage, as many defects immediately anneal after ion impact. The target was set to be hexagonal bulk boron nitride with density of $2.27 \mathrm{~g} / \mathrm{cm}^{2}$ and stoichiometric ratio of $1: 1$. Displacement energies were set to $15 \mathrm{eV}$ for $\mathrm{B}$ and $14 \mathrm{eV}$ for $\mathrm{N}$ [14]. These displacement energies are for the radial direction in the tubes and in reality there is large angular dependence which was not accounted for here.

The damage distribution is shown in Fig. 1. The average values at the area of interest corresponding to nanotube typical diameters $(20-50 \mathrm{~nm})$ are given in Table 1 . It is important to note that in case of $\mathrm{He}$, the Bragg peak is well beyond the nanotube diameters, and the dpa values corresponding to nanotubes are shown in the inset of 1(b). Although the range of $40 \mathrm{keV} \mathrm{Ar}$ is comparable to the tube tickness, damage distribution is relatively uniform from 0 to $50 \mathrm{~nm}$ and one should not expect big differences in morphologies of different nanotubes, as in fact, confirmed by our observations.

3.2 TEM images TEM images of typical irradiated BN nanotubes are presented in Fig. 2. The labeling of the panels is the same as the sample labeling in Table 1.

Panels (a), (b) and (c) show the structure of BN MWNTs after Ar ion irradiation with increasing dose from (a) to (c) (see also Table 1). In panel (a), some interrupted lines can be seen but the tube shells remain mostly intact. Such lines are also present in pristine nanotubes, but their density is normally lower. With increasing the dose, the structure becomes more and more disordered, ending up in nearly amorphous structure for irradiation dose $2 \times 10^{15}$ ions $/ \mathrm{cm}^{2}$. Similar trend was observed for He irradiation, panels (e) and (f), but the difference between intact and irradiated samples was not as big as in the argon case. Some disorder can be seen as darker spots in the panels. However, one should be cautious in the conclusions because of beam heating effects. 

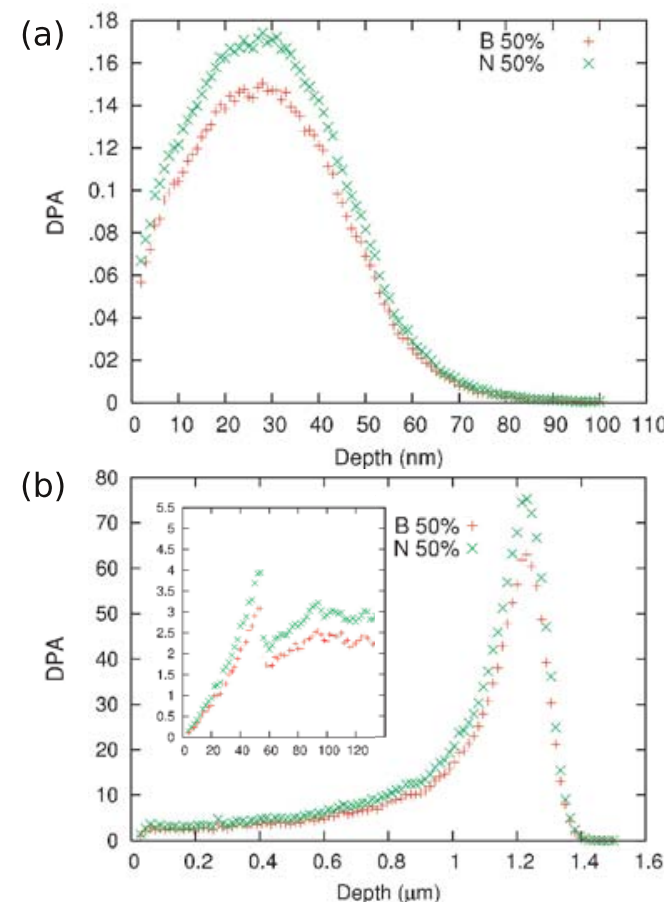

Figure 1 Displacements per atom as a function of depth in boron nitride multi-walled nanotubes subjected to $40 \mathrm{keV}$ argon irradiation with a dose of $1 \times 10^{14}$ ions $/ \mathrm{cm}^{2}$ (a) and $350 \mathrm{keV} \mathrm{He}$ ions with a dose of $1.2 \times 10^{18}$ ions $/ \mathrm{cm}^{2}$ (b). Note the difference in the scales for Ar and He. The inset in panel (b) shows the dpa corresponding to BN MWNTs used in our experiments.

Panels (c) and (d) can be compared in order to assess effects of target temperature during irradiation. The specimen shown in panel (d) was held at elevated temperature of approximately $900 \mathrm{~K}$. Clear difference can be seen between the nanotubes. Elevated temperatures increase the resilience of BN MWNTs to ion irradiation as the number of defects in the heated sample is smaller after similar irradiation doses. This can be attributed to in situ annealing of defects, as in the case of carbon nanotubes, where many defects are repaired almost instantaneously through defect migration and recombination and hence defect accumulation is considerably reduced. This also means that point defects in BN MWNTs are mobile at 900K.

It should be pointed out that due to carbonaceous impurities in the vacuum system during irradiation, some contamination was introduced on the surface of the nanotubes. Such a layer is marked out in panel (f) of Fig. 2. Similar layers are visible in other panels and they can be clearly distinguishable because of the sharp interface. The contamination is composed of mostly carbon, as confirmed by electron energy loss spectroscopy and spatially-resolved elemental mapping. The origin of carbon contamination is not fully understood, but it is very likely that carbonaceous species came from carbon coating of TEM grids due

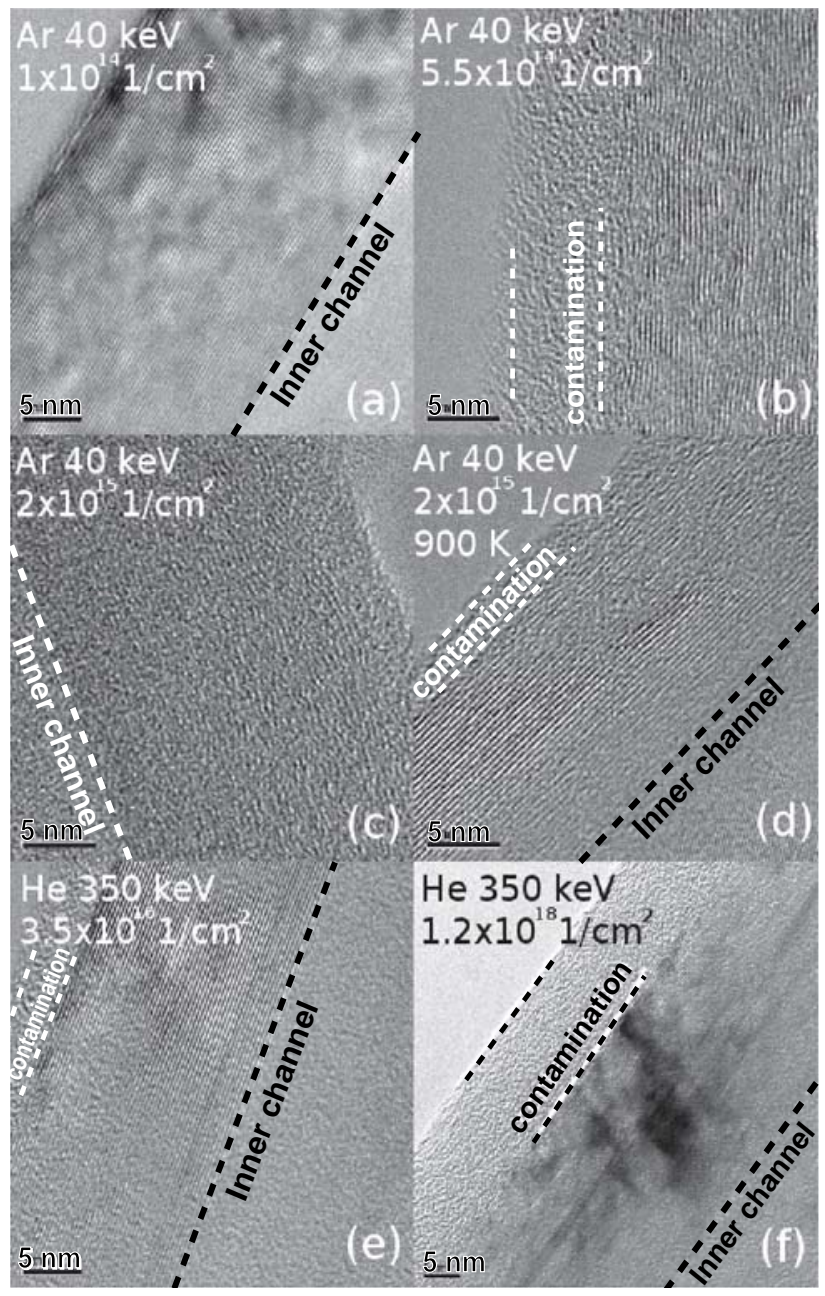

Figure 2 HR-TEM images of the samples with the specification given in Table 1. Only one side of each tube is displayed.

to beam heating and sputtering effects. As $\mathrm{BN}$ nanotubes are non-conducting and thus are charged by the ions, they could have attracted atoms and molecules. Nevertheless, as the thickness of amorphous layer is much lower than projected ranges of either ion type, the contamination does not affect the main conclusions of our work.

The Raman spectra of BN MWNTs irradiated with Ar ions are shown in Fig. 3. The spectra of the carbon coating of TEM grids was subtracted from the measured spectra. Pristine nanotubes exhibit a single peak in the spectra at $1360 \mathrm{~cm}^{-1}$. Irradiation does not change much the main Raman peak. The only difference for the irradiated samples is the appearance of a broad band below $1300 \mathrm{~cm}^{-1}$ at intermediate doses. This band fits the Raman band of BN nanocrystals or BN amorphous phase [20]. The weakness of this feature may be connected with a relative decrease of the Raman scattering cross-section for the disordered phase and with different volumes of ion irradiation and Raman probing (larger for Raman). 


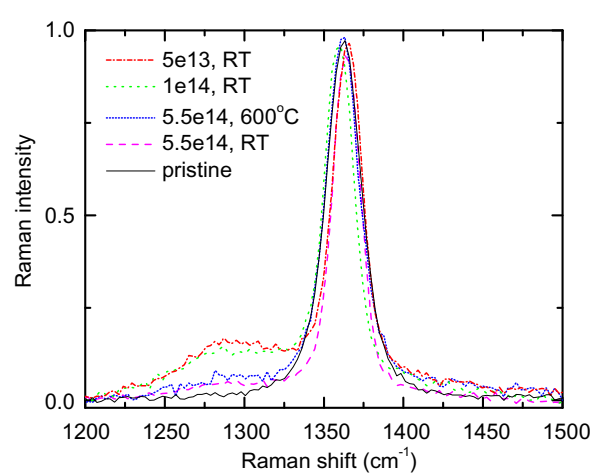

Figure 3 Raman spectra of BN multi-walled nanotubes irradiated with Ar ions. Disorder does not give rise to new peaks, contrary to carbon nanotubes and graphite.

Comparison of panels (c) and (f) can be made to understand contributions of nuclear and electronic stopping mechanisms to defect production. In case of $40 \mathrm{keV} \mathrm{Ar}$ ions the electronic and nuclear stopping power have similar magnitudes, whereas for $350 \mathrm{keV}$ He electronic stopping power is three orders of magnitude larger than nuclear stopping power. Although the values of dpa provided by TRIM are different by a factor of 1.4 , the structure of MWNT, irradiated with Ar ions is much more disordered. Thus one can expect that electronic stopping (at least for values of the stopping power of about $2 \mathrm{MeV} /\left(\mathrm{mg} / \mathrm{cm}^{2}\right)$ ) does not give rise to considerable defect production, but heats the sample, which gives rise to in situ annealing of defects.

Finally, to compare the response of BN and C MWNTs to ion irradiation, we also irradiated C MWNTs. TEM images of the tubes are presented in Fig. 4. For Ar ions, the irradiation doses were the same as for BN MWNTs, for $\mathrm{He}$ ions one order of magnitude less than the maximum dose. The comparison of Fig. 4(a) to Fig. 2(b) and Fig. 4(b) to 4(f) indicates that BN MWNTs appear to be less damaged than their carbon counterparts, as the walls of both $\mathrm{C}$ and $\mathrm{BN}$ pristine nanotubes looked like straight lines in the TEM images.

To sum up, in this paper we presented preliminary results of our experiments on ion irradiation of BN MWNTs. A dose of $2 \times 10^{15}$ ions $/ \mathrm{cm}^{2}$ was found to give rise to complete amorphization for irradiation with $40 \mathrm{keV} \mathrm{Ar}$ ions, while a comparable dose (in terms of displacement per atom) of $1.2 \times 10^{18} \mathrm{ions} / \mathrm{cm}^{2}$ for $350 \mathrm{keV}$ He caused significantly less damage. Raman spectra were found to depend weakly on the number of defects in the sample. Elevated temperatures considerably reduce the amount of damage indicating that efficient annealing of defects occurs in BN nanotubes already at $300^{\circ} \mathrm{C}$. As ion ranges are much higher than the thickness of the contamination layers, our main conclusions should not be affected by the contamination. Overall, our results provide evidence that multi-walled BN nanotubes have similar or even better stability under ion ir-

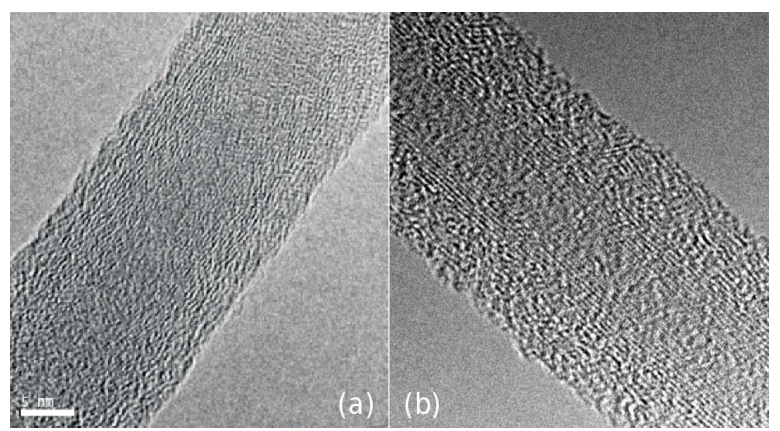

Figure 4 HR-TEM images of carbon multi-walled nanotubes after irradiation with $40 \mathrm{keV} \mathrm{Ar}$ ions and a dose of $5.5 \times 10^{14}$ ions $/ \mathrm{cm}^{2}$ (a). After irradiation with $350 \mathrm{keV} \mathrm{He}$ ions and a dose of $3.5 \times 10^{17}$ ions $/ \mathrm{cm}^{2}(\mathrm{~b})$.

radiation than their carbon counterparts, and our future experiments will assess the relative stability of $\mathrm{BN}$ nanotubes under particular ion beams.

Acknowledgements This work was supported by the Academy of Finland through Centres of Excellence CMS, COMP, and the DEMECAN project and in part by Magnus Ehrnrooth Foundation, and WPI Center for Materials Nanoarchitectonics, NIMS, Japan.

\section{References}

[1] N. G. Chopra et al., Science 269, 966-967 (1995).

[2] D. Golberg et al., Appl. Phys. Lett. 69, 2045-2047 (1996).

[3] D. Golberg et al., Appl. Phys. Lett. 73, 2441-2443 (1998).

[4] D. Pacilé et al., Appl. Phys. Lett. 92, 133107 (2008).

[5] D. Golberg et al., Adv. Mater. 19, 24132432 (2007).

[6] L. Sun et al., Phys. Rev. Lett. 101, 156101 (2008).

[7] A. V. Krasheninnikov et al., Phys. Rev. B 72, 125428 (2005).

[8] A. Celik-Aktas et al., J. Appl. Phys. 102, 024310 (2007).

[9] O. Lehtinen et al., , Physica E 40, 2618-2621 (2008).

[10] A. Colli et al., Nano Letters 8(8), 2188-2193 (2008).

[11] A. V. Krasheninnikov et al., Phys. Rev. Lett. 99, 016104 (2007).

[12] A. V. Krasheninnikov and F. Banhart, Nature Mater. 6, 723-733 (2007).

[13] D. Golberg, unpublished.

[14] A. Zobelli et al., Phys. Rev. B 75, 245402 (2007).

[15] D. Golberg et al. Chem. Phys. Lett. 279, 191 (1997).

[16] J. Ullmann et al., J. Appl. Phys. 83, 2980-2987 (1998).

[17] R. Arenal et al., Nano Lett. 6, 1812 (2006).

[18] L. Khriachtchev, Top. Appl. Phys. 100 (2006) 403. 100, 403 (2006).

[19] Program TRIM (2008) by J.F. Ziegler and J.P. Biersack, http://www.srim.org.

[20] T. Werninghaus et al., Appl. Phys. Lett. 70, 958-960 (1997). 